\title{
PREDIÇÃO DE ESPECTRO DE ATIVIDADES BIOLÓGICAS DA MATRIZ QUÍMICA DO AÇAÍ (EUTERPE OLERACEA MART.) ${ }^{1}$
}

\author{
PREDICTION OF THE BIOLOGICAL ACTIVITIES SPECTRA OF \\ THE CHEMICAL MATRIX OF AÇAÍ (EUTERPE OLERACEA MART.)
}

\author{
Bethina Luiz Dorneles ${ }^{2}$, Lauren Pappis ${ }^{3}$, Diulie Valente de Souza ${ }^{4}$, \\ Gabriela Geraldo Sangoi ${ }^{5}$, Tuyla Fontana ${ }^{5}$ e Alencar Kolinski Machado ${ }^{6}$
}

\section{RESUMO}

Nos dias de hoje, incontáveis estudos sobre produtos de origem natural e seus benefícios são realizados. Nesse contexto, insere-se o açaí (Euterpe oleracea Mart.), um fruto com inúmeras moléculas bioativas que tem um importante papel atribuído ao efeito antioxidante e potencial anti-inflamatório. Devido a isso, é de extrema importância a análise prévia a ensaios in vivo de seus efeitos bioativos cientificamente já identificados, pelo uso de ferramentas computacionais. No presente trabalho, tal avaliação foi realizada por meio de um modelo de estudo de predição de espectro de atividades, através da plataforma PASS Online ${ }^{\circledR}$, na qual a matriz química do açaí foi o objeto de estudo, com a finalidade de reunir dados referentes ao seu metabolismo celular, suas propriedades anti-inflamatórias e suas atividades farmacológicas. Os resultados obtidos em relação ao metabolismo celular oxidativo demonstraram a orientina como o composto que mais se destacou, com elevada capacidade de eliminar radicais livres, mantendo-se acima de $90 \%$, e maior potencial antioxidante, que permaneceu acima de $80 \%$. No que diz respeito às propriedades anti-inflamatórias, sobressaíram-se o ácido cafeico e o ácido $p$-cumárico; ambos se mantiveram acima de $70 \%$, quando avaliados quanto ao seu efeito inibidor de fator de necrose tumoral. Ainda, no que tange às questões farmacológicas, suas atividades atuantes nos cânceres chamam a atenção: o potencial anticarcinogênico e o antineoplásico apresentaram compostos com efeitos acima de $80 \%$, enquanto o potencial antimutagênico superou $90 \%$. Conclui-se que, além de o açaí ser uma excelente alternativa a estudos que buscam pelo desenvolvimento de novos métodos terapêuticos, seus efeitos bioativos se devem à variedade de moléculas encontradas em sua matriz química.

Palavras-chave: Compostos orgânicos, Efeitos bioativos, Moléculas bioativas, Produtos naturais.

\section{ABSTRACT}

Nowadays, it is known that countless studies on products of natural origin and their benefits are carried out. In this context, açai (Euterpe oleracea Mart.) Is inserted, a fruit with numerous bioactive molecules and an important role attributed to the antioxidant effect and anti-inflammatory potential. Because of this, it's extremely important to analyze prior to in vivo tests its bioactive effects scientifically identified, using computational tools. In the present work, such an assessment was made using a prediction of activities spectra

1 Trabalho Final de Graduação.

2 Autor. Curso de Biomedicina - Universidade Franciscana. E-mail: dornelesbethina@gmail.com

3 Co-autor. Programa de Pós-Graduação em Ciências Farmacêuticas - Universidade Federal de Santa Maria. E-mail: laurenpappis@gmail.com

4 Co-autor. Programa de Pós-Graduação em Nanociências - Universidade Franciscana. E-mail: diully_desouza@ hotmail.com

5 Co-autor. Curso de Biomedicina - Universidade Franciscana. E-mail: gabriela.sangoi30@gmail.com; tuyla_fontana@ hotmail.com

6 Orientador. Docente do curso de Biomedicina - Universidade Franciscana. E-mail: alencarkolinski@gmail.com 
study model, through the PASS Online ${ }^{\circledR}$ platform, where the chemical matrix of açai was the object of study, with the purpose of gathering data related to its cellular metabolism, its anti-inflammatory diseases and their pharmacological activities. The results obtained in relation to oxidative cell metabolism demonstrated orientin as the compound that stood out the most, with a high capacity to eliminate free radicals, remaining above $90 \%$, and greater antioxidant potential, which remained above $80 \%$. Regarding the anti-inflammatory properties, caffeic acid and p-cumaric acid stood out and both remained above 70\%, when evaluated for their inhibitory effect on tumor necrosis factor. Still, in relation to pharmacological issues, their activities in cancers call attention: anticarcinogenic and antineoplastic potential presented compounds with effects above $80 \%$ and the antimutagenic potential exceeded $90 \%$. It's concluded that açai is an excellent alternative to studies that seek to develop new therapeutic methods and their bioactive effects are due to the variety of molecules found in its chemical matrix.

Keywords: Organic compounds, Bioactive effects, Bioactive molecules, Natural products.

\section{INTRODUÇÃO}

Inúmeras foram as transformações que ocorreram em meados do século XX e que vêm se potencializando, atualmente, na área da saúde. Isso se deve por conta das evoluções tecnológicas que acarretaram várias mudanças de cunho político, econômico, social e cultural, interferindo diretamente no modo de vida da população. Todavia, apesar de vários avanços positivos, existem também pontos negativos que acarretam, por exemplo, no surgimento e desenvolvimento de doenças de complexa fisiopatologia em maior escala (MINISTÉRIO DA SAÚDE, 2014).

Além disso, considerando a terapêutica de tais doenças, em algumas situações, os métodos de tratamento existentes acabam não possuindo finalidade curativa, mas atuando de forma a amenizar sinais e sintomas (ADÃO et al., 2013; MACHADO et al., 2016; MANCINI, 2001). Dentre as doenças que ainda instigam profissionais pesquisadores a uma melhor compreensão fisiopatológica, estão: câncer (NUSSBAUM et al., 2008; FERLAY et al., 2015), doenças neuropsiquiátricas (ANDREAZZA et al., 2013; MACHADO et al., 2016), doenças metabólicas (BOCKENHAUER; BICHET, 2015) e até mesmo infecções virais (WANG et al., 2020).

Por outro lado, a qualidade dos alimentos funcionais tem sido alvo de estudos em todo o mundo, não apenas por seu valor nutricional, mas também pelos efeitos bioativos que eles exercem em um organismo vivo. Sobre tais efeitos, há evidências científicas de produtos naturais que atuam, por exemplo, como antioxidantes (SAGRILLO et al., 2015), antitumorais (CADONÁ et al., 2017), anti-inflamatórios (DE SOUZA et al., 2020), ou mesmo como neuroprotetores (MACHADO et al., 2016). Vale ressaltar que esses efeitos são associados à composição da matriz química de tais produtos, pois incluem a presença de diferentes moléculas bioativas. Logo, neste campo de investigação, o uso de elementos naturais e seus derivados vem se destacando tanto no sentido de eficácia, quanto de segurança (SAGRILLO et al., 2015; CADONÁ et al., 2016; NEWMAN; CRAGG, 2016; LAJOLO, 2002). 
Pesquisadores de vários continentes buscam, cada vez mais, alternativas naturais que possam ser inseridas no exercício da medicina tradicional. Até o momento, muitos foram os avanços quanto a tratamentos e intervenções terapêuticas e de prevenção de doenças que possuem grande impacto à saúde pública mundial, bem como para doenças mais raras que afetam uma parcela mais reduzida da população (GRUNZE et al., 2010).

Dentre os produtos naturais que têm ganhado destaque em pesquisas científicas, pode-se citar o guaraná (MACHADO et al., 2014; CADONÁ et al., 2016; 2017), o butiá (FONSECA, 2011; HARBORNE; WILLIAMS, 2000), o tucumã (SAGRILLO et al., 2015) e o açaí (MACHADO et al., 2016; 2019; DE SOUZA et al., 2020), sendo este último o objeto de estudo desta investigação. O açaí (Euterpe oleracea Mart.) é um fruto oriundo da região amazônica do Brasil, altamente consumido no país e que tem ganhado o mercado internacional. A polpa apresenta em sua composição alto valor energético com lipídeos (Ômegas 6 e 9), carboidratos, fibras, vitamina E, proteínas e minerais (ferro, zinco, cobre, manganês e crômio). Em suma, possui uma matriz química rica em moléculas com efeitos bioativos, como antioxidante, analgésico e neuroprotetor, que são capazes de interagir positivamente com as células do metabolismo celular oxidativo, além de atuar no perfil imunológico, em dislipidemias, cânceres e no envelhecimento (PORTINHO; ZIMMERMANN; BRUCK, 2012; MACHADO et al., 2016).

Nesse contexto, a literatura traz estudos que mostram efeitos promissores do açaí. A exemplo disso, Machado e colaboradores (2016) demonstraram que o extrato hidroalcoólico de açaí é capaz de induzir um efeito nutrigenômico em neurônios com disfunção mitocondrial, provocada pela exposição à rotenona. Dessa forma, o extrato foi capaz de recuperar a atividade do complexo mitocondrial I, reduzindo os efeitos deletérios do estresse oxidativo.

Ademais, já se tem conhecimento de que o açaí possui potencial anti-inflamatório. Machado e colaboradores (2019), por exemplo, sugeriram tal efeito em macrófagos induzidos à inflamação com fitohemaglutinina, evidenciando que tal efeito se deve à modulação do inflamassoma NLRP3. Ao encontro disso, de Souza et al. (2020) também apontaram o efeito anti-neuroinflamatório in vitro em micróglias ativadas com lipopolissacarídeo (LPS).

Contudo, apesar de vários estudos envolvendo o açaí, ainda não há relatos de uma análise de predição complementar que confirme os efeitos já descritos, bem como exponha novas propriedades desse fruto através de uma avaliação computacional. Logo, os métodos predição podem ser uma excelente ferramenta para, além de permitir a exploração de novas propriedades das moléculas avaliadas, predizer potenciais relações entre as estruturas e as atividades biológicas de tais constituintes. Levando isso em consideração, este trabalho busca realizar uma análise de predição de espectro de atividades biológicas da matriz química de um extrato hidroalcoólico de açaí, utilizando recurso computacional online. 


\section{MATERIAIS E MÉTODOS}

Os dados da caracterização do extrato hidroalcoólico de Euterpe oleracea Mart. foram obtidos por meio do estudo de Machado e colaboradores (2016), no qual tal extrato foi submetido à cromatografia líquida de alta eficiência (HPLC), conforme descrito por Boligon e colaboradores (2015). A concentração do extrato para caracterização foi de $10 \mathrm{mg} / \mathrm{mL}$; durante o processo foram utilizados o aparelho modelo SIL-20A Shimadzu Auto Sampler ${ }^{\circledR}$ e a coluna de separação Phenomenex $C_{18}{ }^{\circledR}$.

As quantificações da matriz química, por sua vez, foram conduzidas pela integração dos picos, com base em padrões externos, adquiridos por intermédio da empresa Sigma-Aldrich ${ }^{\circledR}$ (São Paulo, SP, Brasil). Os picos cromatográficos foram confirmados a partir da comparação entre os tempos de retenção e a variável dos padrões descritos. Os componentes encontrados na matriz química do extrato do açaí foram: ácido gálico, catequina, ácido clorogênico, ácido cafeico, ácido p-cumárico, epicatequina, cianeto-3-0-glucosídeo, orientina, luteolina e apigenina (figura 1).

Figura 1 - Estrutura química das moléculas presentes no extrato hidroalcoólico de açaí.

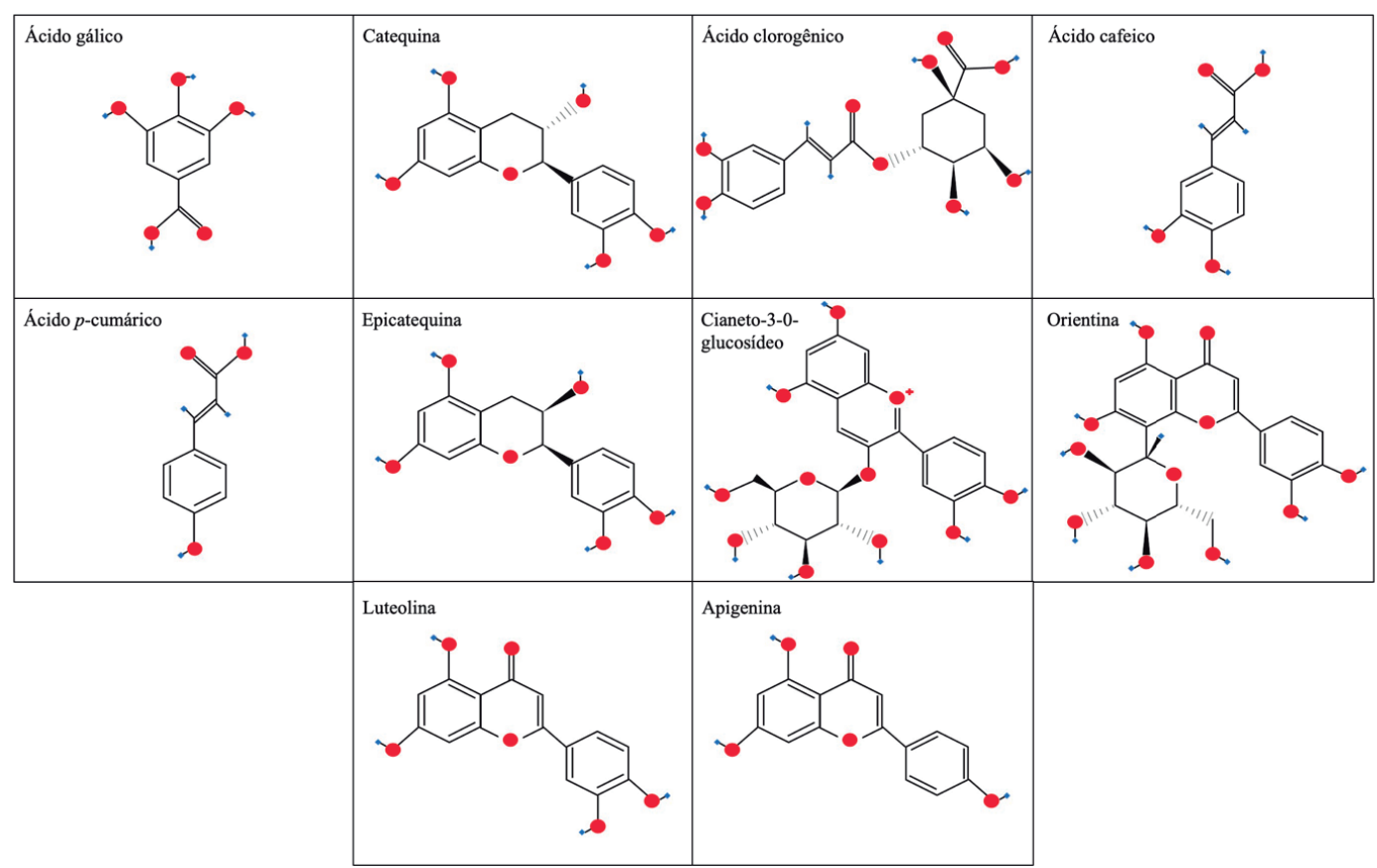

Fonte: elaborado pelos autores (2021). As imagens foram criadas no programa Microsoft Power Point, versão 2010, com base nas estruturas apresentadas na plataforma PubChem ${ }^{\circledR}$. Os pontos vermelhos representam Oxigênio $(\mathrm{O})$ e os pontos azuis, Hidrogênio $(\mathrm{H})$.

O estudo de predição de espectro de atividades biológicas foi realizado através do banco de dados PubChem ${ }^{\circledR}$ do National Institutes of Health (NIH) (https://pubchem.ncbi.nlm.nih.gov/) e do software gratuito PASS Online ${ }^{\circledR}$ (Prediction of Activity Spectra for Substances) do portal Way2Drug (http://www.way2drug.com/PassOnline/). O banco de dados favoreceu a obtenção da forma canonical SMILE das moléculas do extrato hidroalcoólico do açaí, e o software entregou previsões simultâneas de muitos tipos de atividade biológica, com base na estrutura dos compostos orgânicos. 
A plataforma $P A S S$ provê o espectro de atividade biológica de um composto químico, onde o conjunto de diferentes tipos de atividade biológica refletem os resultados da interação do composto com várias entidades biológicas, possibilitando estimar o potencial de atividade de uma substância, sendo classificadas como Pa (probabilidade do composto "ser ativo") e Pi (probabilidade do composto "ser inativo"), descritos em porcentagem. A plataforma representa a propriedade "intrínseca" de uma substância, dependendo apenas de sua estrutura e características físico-químicas e possui a possibilidade de combinação de várias fontes diferentes no mesmo conjunto, já que apenas uma publicação não consegue abranger todas as características de ação biológica de compostos.

Os compostos orgânicos foram avaliados conforme seu metabolismo oxidativo celular (inibidor da permeabilidade de membrana, eliminador de radicais livres, inibidor da peroxidase lipídica, inibidor da enzima oxirredutase, antioxidante, inibidor da expressão de óxido nítrico sintase-2 (NOS2) e antagonista de óxido nítrico), potencial anti-inflamatório (estimulante de enzima caspase 3, estimulante da enzima caspase 8, estimulante do fator de transcrição NF kappa B, inibidor da expressão de fator de necrose tumoral (TNF), inibidor da enzima ATPase, antagonista de Interferon gamma) e atividades farmacológicas (radioprotetor, antimutagênico, vasoprotetor, retinoprotetor, estimulante de eritropoiese, anticarcinogênico, quimiopreventivo, imunossupressor, antineoplásico, antifúgico, anti-inflamatório, hepatoprotetor, antimetastático e citoprotetor).

\section{RESULTADOS E DISCUSSÃO}

\section{METABOLISMO CELULAR OXIDATIVO}

Em relação ao metabolismo celular oxidativo dos compostos encontrados na matriz química do açaí, pode-se observar relevantes efeitos bioativos (Tabela 1). Em todos eles, a probabilidade de ser ativa $(\mathrm{Pa})$ se mostrou superior à probabilidade de ser inativa $(\mathrm{Pi})$.

A orientina, molécula majoritária da matriz química do extrato de açaí, mostrou elevada capacidade de eliminar radicais livres (Pa: 0,955 / Pi: 0,001), e o maior potencial antioxidante (Pa: 0,828 / Pi: 0,003). Lobo e Velasque (2016), em uma revisão de literatura, sinalizam que flavonoides, como a orientina, são capazes de capturar radicais livres que estão à procura de alvos celulares para corrigirem o seu desemparelhamento de elétrons, interferindo diretamente no processo de estresse oxidativo. Por fim, Cadoná e colaboradores (2021) demonstram o potencial efeito antioxidante in vitro da orientina, utilizando tal molécula como um controle positivo em um modelo.

Além disso, o produto da ação da enzima peroxidase lipídica é responsável pela formação de compostos instáveis e, como resultado positivo, a maioria dos compostos encontrados na matriz química do açaí mostraram expressivos resultados quanto à capacidade de inibição dessa enzima. 
Em uma revisão da literatura, Galleano, Pechanova e Fraga (2010) enfatizam que os polifenóis, incluindo catequina e epicatequina, possuem capacidade de atenuar a hipertensão, especialmente através da redução de óxido nítrico. Somando a isso, Gentile e colaboradores (2018) também observaram que a luteolina é capaz de modular a produção de óxido nítrico, no sentido de reduzir os níveis de tal molécula em camundongos. Ainda, em um estudo realizado por Machado e colaboradores (2019), os autores descrevem que o extrato hidroalcoólico de açaí foi capaz de reduzir significativamente os níveis excessivos de óxido nítrico. De modo geral, essas pesquisas in vitro corroboraram com os achados aqui descritos.

O óxido nítrico é uma molécula instável, com tempo de meia-vida curto, que age como mensageira, sendo responsável por mediar diversas funções biológicas, como resposta inflamatória. Em altas concentrações, esse composto pode ser prejudicial ao corpo humano. Conforme descrito (Tabela 1), os compostos químicos do açaí que apresentaram maior efeito antagonista ao óxido nítrico, ou seja, que bloquearam a ação dele, foram: catequina (Pa: 0,541 / Pi: 0,004), epicatequina (Pa: 0,541 / Pi: 0,004) e luteolina (Pa: 0,551 / Pi: 0,004).

Dessa forma, percebe-se que neste parâmetro de avaliação, tais valores podem ser considerados menos expressivos, quando comparados aos estudos citados anteriormente que mostram a redução da produção dos níveis excessivos de óxido nítrico, já que todos os resultados se mantiveram em torno de $50 \%$.

Tabela 1 - Avaliação do espectro de predição da modulação de parâmetros do metabolismo oxidativo celular pela matriz química do extrato de açaí.

\begin{tabular}{|c|c|c|c|c|c|c|c|c|c|c|c|}
\hline Matriz Química & & 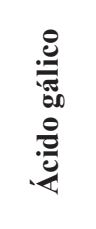 & 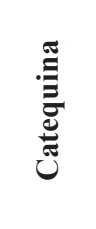 & 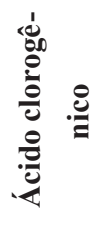 & 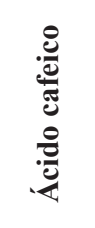 & 旡 & 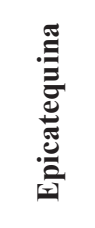 & & 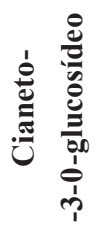 & 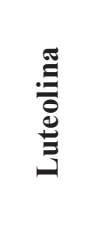 & 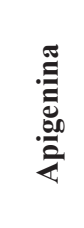 \\
\hline \multirow{2}{*}{$\begin{array}{l}\text { Inibidor permeabilidade } \\
\text { de membrana }\end{array}$} & $\mathrm{Pa}$ & 0,815 & 0,790 & 0,865 & 0,809 & 0,809 & 0,790 & 0,904 & ND & 0,953 & 0,946 \\
\hline & $\mathrm{Pi}$ & 0,008 & 0,011 & 0,004 & 0,009 & 0,009 & 0,011 & 0,004 & ND & 0,002 & 0,002 \\
\hline \multirow{2}{*}{$\begin{array}{l}\text { Eliminador de } \\
\text { radicais livres }\end{array}$} & $\mathrm{Pa}$ & 0,570 & 0,842 & 0,856 & 0,647 & 0,627 & 0,842 & 0,955 & ND & 0,755 & 0,719 \\
\hline & $\mathrm{Pi}$ & 0,007 & 0,002 & 0,002 & 0,005 & 0,005 & 0,002 & 0,001 & ND & 0,003 & 0,004 \\
\hline \multirow{2}{*}{$\begin{array}{l}\text { Inibidor da } \\
\text { peroxidase lipídica }\end{array}$} & $\mathrm{Pa}$ & 0,554 & 0,888 & 0,855 & 0,570 & 0,529 & 0,888 & 0,760 & ND & 0,740 & 0,695 \\
\hline & $\mathrm{Pi}$ & 0,012 & 0,003 & 0,003 & 0,011 & 0,014 & 0,003 & 0,004 & ND & 0,004 & 0,005 \\
\hline \multirow{2}{*}{$\begin{array}{l}\text { Inibidor da } \\
\text { oxirredutase }\end{array}$} & $\mathrm{Pa}$ & 0,717 & $\mathbf{0 , 5 7 1}$ & 0,846 & 0,654 & 0,667 & $\mathbf{0 , 5 7 1}$ & 0,578 & ND & 0,693 & 0,692 \\
\hline & $\mathrm{Pi}$ & 0,013 & 0,047 & 0,004 & 0,023 & 0,020 & 0,047 & 0,044 & ND & 0,016 & 0,016 \\
\hline \multirow{2}{*}{ Antioxidante } & $\mathrm{Pa}$ & 0,520 & 0,810 & 0,785 & 0,603 & 0,553 & 0,810 & 0,828 & ND & 0,755 & 0,732 \\
\hline & $\mathrm{Pi}$ & 0,006 & 0,003 & 0,004 & 0,005 & 0,005 & 0,003 & 0,003 & ND & 0,004 & 0,004 \\
\hline \multirow{2}{*}{$\begin{array}{l}\text { Inibidor da } \\
\text { expressão de NOS2 }\end{array}$} & $\mathrm{Pa}$ & 0,382 & 0,631 & $\mathbf{0 , 3 3 1}$ & 0,508 & 0,490 & 0,631 & 0,559 & ND & 0,798 & 0,732 \\
\hline & $\mathrm{Pi}$ & 0,022 & 0,003 & 0,033 & 0,008 & 0,010 & 0,003 & 0,005 & ND & 0,002 & 0,002 \\
\hline \multirow{2}{*}{$\begin{array}{l}\text { Antagonista de } \\
\text { óxido nítrico }\end{array}$} & $\mathrm{Pa}$ & 0,291 & 0,541 & 0,266 & 0,338 & 0,310 & 0,541 & 0,420 & ND & $\mathbf{0 , 5 5 1}$ & 0,444 \\
\hline & $\mathrm{Pi}$ & 0,015 & 0,004 & 0,016 & 0,010 & 0,013 & 0,004 & 0,006 & ND & 0,004 & 0,006 \\
\hline
\end{tabular}


Quanto ao efeito das diferentes moléculas da matriz química do extrato de açaí sobre o sistema inflamatório, foram avaliados diferentes parâmetros vinculados à ativação inflamatória, incluindo diferentes caspases, citocinas pró-inflamatórias e a ATPase, já que tais moléculas estão intimamente vinculadas à cascata inflamatória.

As caspases são um grupo de proteases relacionadas à morte programada das células. A caspase 3 está diretamente ligada aos efeitos celulares deletérios da apoptose (CROWLEY; WATERHOUSE, 2016). Como a Tabela 2 ilustra, catequina e epicatequina mostraram resultados significativos em relação à estimulação de caspase 3, onde: catequina (Pa: 0,661 / Pi: 0,013) e epicatequina (Pa: 0,661 / Pi: 0,013). Esses resultados estão em conformidade ao estudo de Del Pozo-Insfran, Percival e Talcott (2006), que sugere que a apoptose induzida pelo açaí envolve um mecanismo mediado pela caspase 3, capaz de reduzir a proliferação in vitro de células leucêmicas HL-60, por exemplo.

O inibidor de TNF é outro parâmetro que merece atenção nesta avaliação. O TNF possui uma atividade necrotizante contra linhagens de células (DeCS, 2017). Entretanto, sua produção excessiva resulta em uma maior liberação da cascata de inflamação, podendo acarretar em um processo citotóxico. Por esse motivo, é preciso que se tenha controle da produção de fator de necrose tumoral, através de inibidores de TNF. Como mostrado na tabela 2, no que concerne à inibição da expressão de TNF, ácido cafeico e ácido p-cumárico são os que mais se destacam (Pa: 0,765 / Pi: 0,004 e Pa: 0,737 / Pi: 0,005, respectivamente). Tal fato está de acordo com o estudo ex vivo de Lima (2017), no qual a autora demonstra que o uso do extrato do açaí pode ser uma estratégia protetora adicional, pois é capaz de suprimir a sinalização de mediadores inflamatórios, como o TNF alfa, em células mesangiais de camundongos.

Já o Interferon gamma (IFN- $\gamma$ ) é uma citocina pró-inflamatória envolvida nas respostas imunes dirigidas por células Th1. Acerca do parâmetro de avaliação "Antagonista de Interferon gamma", percebe-se uma baixa nos valores: todos se mantiveram abaixo de $20 \%$. Por outro lado, Machado e colaboradores (2019), em um estudo in vitro desenvolvido com macrófagos ativados, observaram que o extrato de açaí pode modular citocinas pró-inflamatórias, inclusive o IFN- $\gamma$. Acredita-se que esta modulação se deva a um efeito sinérgico entre as moléculas presentes na matriz química do extrato. 
Tabela 2 - Avaliação do potencial anti-inflamatório da matriz química do extrato de açaí.

\begin{tabular}{|c|c|c|c|c|c|c|c|c|c|c|c|}
\hline Matriz Química & & 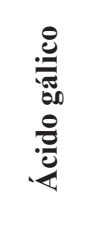 & שֶّ & 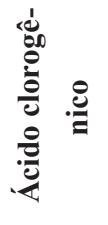 & 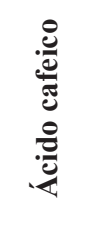 & 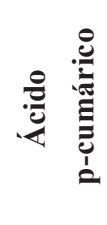 & 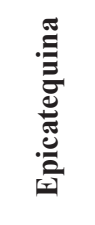 & & 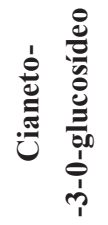 & 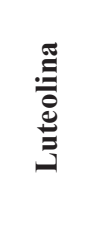 & 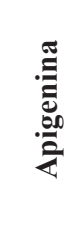 \\
\hline \multirow{2}{*}{ Estimulante da caspase 3} & $\mathrm{~Pa}$ & 0,413 & 0,661 & 0,599 & 0,579 & 0,496 & 0,661 & $\mathbf{0 , 5 8 7}$ & ND & 0,632 & $\mathbf{0 , 5 7 7}$ \\
\hline & $\mathrm{Pi}$ & 0,046 & 0,013 & 0,017 & 0,018 & 0,028 & 0,013 & 0,018 & ND & 0,015 & 0,019 \\
\hline \multirow{2}{*}{ Estimulante da caspase 8} & $\mathrm{~Pa}$ & 0,492 & 0,469 & 0,534 & 0,495 & 0,438 & 0,469 & 0,601 & ND & 0,480 & 0,450 \\
\hline & $\mathrm{Pi}$ & 0,019 & 0,024 & 0,012 & 0,018 & 0,034 & 0,024 & 0,007 & ND & 0,022 & 0,030 \\
\hline \multirow{2}{*}{$\begin{array}{l}\text { Estimulante do fator de } \\
\text { transcrição NF kappa B }\end{array}$} & $\mathrm{Pa}$ & 0,388 & 0,302 & 0,524 & 0,458 & 0,501 & 0,302 & 0,422 & ND & 0,278 & 0,280 \\
\hline & $\mathrm{Pi}$ & 0,057 & 0,110 & 0,018 & 0,033 & 0,022 & 0,110 & 0,044 & ND & 0,134 & 0,132 \\
\hline \multirow{2}{*}{$\begin{array}{l}\text { Inibidor da } \\
\text { expressão de TNF }\end{array}$} & $\mathrm{Pa}$ & 0,560 & $\mathbf{0 , 5 1 7}$ & 0,519 & 0,765 & 0,737 & 0,517 & 0,345 & ND & 0,635 & 0,609 \\
\hline & $\mathrm{Pi}$ & 0,018 & 0,026 & 0,025 & 0,004 & 0,005 & 0,026 & 0,077 & ND & 0,010 & 0,012 \\
\hline \multirow{2}{*}{ Inibidor de ATPase } & $\mathrm{Pa}$ & 0,344 & 0,186 & 0,427 & 0,297 & 0,273 & 0,186 & 0,207 & ND & 0,275 & 0,286 \\
\hline & $\mathrm{Pi}$ & 0,007 & 0,093 & 0,004 & 0,014 & 0,021 & 0,093 & 0,069 & ND & 0,020 & 0,016 \\
\hline Antagonista de & $\mathrm{Pa}$ & 0,199 & 0,138 & 0,231 & 0,176 & 0,188 & 0,138 & 0,150 & ND & 0,144 & 0,148 \\
\hline Interferon gamma & $\mathrm{Pi}$ & 0,017 & 0,056 & 0,012 & 0,025 & 0,020 & 0,056 & 0,044 & ND & 0,050 & 0,046 \\
\hline
\end{tabular}

Potencial de ser ativo $>50 \%$ : AZUL Potencial de ser ativo $<50 \%$ : VERMELHO

\section{ATIVIDADES FARMACOLÓGICAS}

Em relação aos cânceres, a presente investigação se mostrou de grande valia. Como descrito na Tabela 3, observou-se o potencial anticarcinogênico através do ácido clorogênico (Pa: 0,846 / Pi: 0,004) e da orientina (Pa: 0,872 / Pi: 0,003), além do efeito antineoplásico por meio da orientina (Pa: 0,844 / Pi: 0,007). Esta análise corresponde ao que foi constatado por Portinho, Zimmermann e Bruck (2017) que, após estudos in vitro, foram capazes de demonstrar que frações polifenólicas da polpa do açaí eram capazes de reduzir a proliferação das células leucêmicas HL-60 e atenuar a proliferação celular e o tamanho dos tumores.

Outrossim, o efeito antimutagênico foi mais um parâmetro usado nesta avaliação. No que se refere à luteolina, pertencente à família dos flavonoides, conhecidos por suas diversas atividades biológicas, o trabalho in vitro de Resende (2011) foi capaz de demonstrar um efeito protetor contra todos os mutágenos testados, exibindo, dessa maneira, atividade antimutagênica de moderada a forte, consoante ao que foi descrito pela plataforma PASS Online ${ }^{\circledR}$ : Pa de 0,940 e Pi de 0,001. Isso indica um passo positivo na determinação do uso seguro desses flavonoides.

Ainda, há o efeito quimiopreventivo. Um estudo realizado em modelo in vivo de endometriose em ratos por Baptista (2018) sugeriu que o açaí pudesse ser utilizado como agente seguro e quimiopreventivo. Nesse estudo, ácido clorogênico e orientina foram os compostos com números mais expressivos, quando relacionados à utilização de agentes químicos naturais ou sintéticos na reversão, bloqueio ou prevenção do surgimento do câncer, com Pa: 0,833 / Pi: 0,003 e Pa: 0,881 / Pi: 0,003, respectivamente. 
Além disso, outras propriedades farmacológicas foram encontradas, a exemplo da luteolina e da apigenina que mais se destacaram como vasoprotetores (Pa: 0,901 / Pi: 0,003, Pa: 0,891 / Pi: 0,003, respectivamente). Substâncias vasoprotetoras melhoram a circulação sanguínea no corpo humano, aumentando a oxigenação e, por isso, são de extrema importância para auxiliar em zumbidos, labirintites, edemas, varizes e até mesmo câimbras. Rocha e colaboradores (2007), via um estudo in vivo, sugerem que o efeito vasodilatador pode ser alcançado com o uso do açaí, podendo ser inserido no tratamento desses problemas, assim como em doenças cardiovasculares.

Por fim, a orientina demonstrou alta taxa de capacidade hepatoprotetora (Pa: 0,927 / Pi: 0,002). Tal fato vai ao encontro das comprovações de um estudo conduzido com modelo de camundongos alimentados com uma dieta rica em gorduras e que possuíam adiposidade, obesidade e esteatose hepática, por De Oliveira e colaboradores (2015). Os pesquisadores constataram que um extrato de semente de Euterpe oleracea Mart. foi capaz de reduzir os níveis de glicose, o acúmulo de colesterol e os triglicerídeos no fígado, além de uma redução de ganho de peso corporal; tudo isso estava associado a uma redução da esteatose. Em síntese, o efeito antioxidante do extrato foi demonstrado pela restauração das atividades das enzimas superóxido dismutase (SOD), catalase (CAT) e glutationa peroxidase (GPx) e pela redução dos níveis aumentados de malondialdeído e carbonilação de proteínas, melhorando o estresse oxidativo hepático.

Tabela 3 - Avaliação das atividades farmacológicas da matriz química do extrato de açaí.

\begin{tabular}{|c|c|c|c|c|c|c|c|c|c|c|c|}
\hline Matriz Química & & 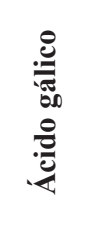 & لَّ & 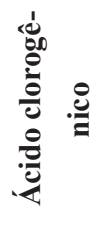 & 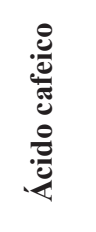 & 兽 & 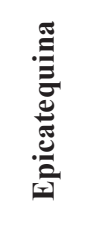 & & 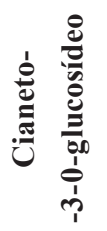 & 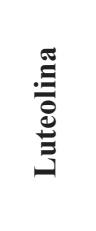 & 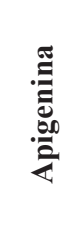 \\
\hline \multirow{2}{*}{ Radioprotetor } & P.a & 0,620 & 0,402 & 0,469 & 0,611 & 0,573 & 0,402 & 0,616 & ND & 0,567 & 0,564 \\
\hline & P.i & 0,014 & 0,043 & 0,029 & 0,015 & 0,018 & 0,043 & 0,014 & ND & 0,019 & 0,019 \\
\hline \multirow{2}{*}{ Antimutagênico } & P.a & 0,597 & 0,571 & 0,409 & 0,845 & 0,886 & 0,571 & 0,835 & ND & 0,940 & 0,921 \\
\hline & P.i & 0,010 & 0,011 & 0,022 & 0,003 & 0,002 & 0,011 & 0,003 & ND & 0,001 & 0,002 \\
\hline \multirow{2}{*}{ Vasoprotetor } & P.a & 0,565 & 0,652 & 0,442 & 0,782 & 0,735 & 0,652 & 0,884 & ND & 0,901 & 0,891 \\
\hline & P.i & 0,025 & 0,014 & 0,059 & 0,006 & 0,008 & 0,014 & 0,003 & ND & 0,003 & 0,003 \\
\hline \multirow{2}{*}{ Retinoprotetor } & P.a & 0,297 & 0,233 & 0,324 & 0,305 & 0,302 & 0,233 & 0,222 & ND & ND & 0,216 \\
\hline & P.i & 0,032 & 0,148 & 0,018 & 0,027 & 0,028 & 0,148 & 0,192 & ND & ND & 0,214 \\
\hline \multirow{2}{*}{$\begin{array}{l}\text { Estimulante de eritro- } \\
\text { poiese }\end{array}$} & P.a & 0,700 & 0,299 & 0,367 & 0,600 & 0,624 & 0,299 & ND & ND & 0,377 & 0,432 \\
\hline & P.i & 0,005 & 0,182 & 0,113 & 0,013 & 0,010 & 0,182 & ND & ND & 0,105 & 0,069 \\
\hline \multirow{2}{*}{ Anticarcinogênico } & P.a & 0,395 & 0,795 & 0,846 & 0,571 & 0,559 & 0,795 & 0,872 & ND & 0,690 & 0,641 \\
\hline & P.i & 0,031 & 0,005 & 0,004 & 0,014 & 0,015 & 0,005 & 0,003 & ND & 0,009 & 0,011 \\
\hline \multirow{2}{*}{ Quimiopreventivo } & P.a & 0,406 & 0,788 & 0,833 & 0,551 & 0,516 & 0,788 & 0,881 & ND & 0,648 & 0,593 \\
\hline & P.i & 0,022 & 0,004 & 0,003 & 0,012 & 0,014 & 0,004 & 0,003 & ND & 0,008 & 0,010 \\
\hline \multirow{2}{*}{ Imunossupressor } & P.a & 0,351 & 0,348 & 0,700 & 0,379 & 0,401 & 0,348 & 0,509 & ND & 0,243 & 0,252 \\
\hline & P.i & 0,084 & 0,086 & 0,017 & 0,071 & 0,065 & 0,086 & 0,041 & ND & 0,153 & 0,147 \\
\hline \multirow{2}{*}{ Antineoplásico } & P.a & 0,313 & 0,675 & 0,778 & 0,530 & 0,520 & 0,675 & 0,844 & ND & 0,783 & 0,774 \\
\hline & P.i & 0,145 & 0,030 & 0,014 & 0,063 & 0,065 & 0,030 & 0,007 & ND & 0,014 & 0,015 \\
\hline \multirow{2}{*}{ Antifúgico } & P.a & 0,398 & 0,552 & 0,638 & 0,450 & 0,451 & 0,552 & 0,714 & ND & 0,520 & 0,524 \\
\hline & P.i & 0,050 & 0,023 & 0,014 & 0,039 & 0,039 & 0,023 & 0,009 & ND & 0,027 & 0,027 \\
\hline
\end{tabular}




\begin{tabular}{lccccccccccc} 
Anti-inflamatório & P.a & $\mathbf{0 , 5 4 8}$ & $\mathbf{0 , 5 4 8}$ & $\mathbf{0 , 5 9 8}$ & $\mathbf{0 , 6 5 1}$ & $\mathbf{0 , 6 4 1}$ & $\mathbf{0 , 5 4 8}$ & $\mathbf{0 , 6 2 6}$ & ND & $\mathbf{0 , 6 6 1}$ & $\mathbf{0 , 6 4 4}$ \\
& P.i & 0,044 & 0,044 & 0,032 & 0,023 & 0,024 & 0,044 & 0,027 & ND & 0,021 & 0,024 \\
\hline \multirow{3}{*}{ Hepatoprotetor } & P.a & $\mathbf{0 , 5 0 4}$ & $\mathbf{0 , 6 7 9}$ & $\mathbf{0 , 5 8 4}$ & $\mathbf{0 , 4 6 1}$ & $\mathbf{0 , 5 5 3}$ & $\mathbf{0 , 6 7 9}$ & $\mathbf{0 , 9 2 7}$ & ND & $\mathbf{0 , 6 5 8}$ & $\mathbf{0 , 6 2 7}$ \\
& P.i & 0,020 & 0,008 & 0,014 & 0,024 & 0,016 & 0,008 & 0,002 & ND & 0,009 & 0,010 \\
\multirow{3}{*}{ Antimetastático } & P.a & ND & $\mathbf{0 , 2 9 0}$ & $\mathbf{0 , 5 4 2}$ & $\mathbf{0 , 4 4 3}$ & $\mathbf{0 , 4 6 4}$ & $\mathbf{0 , 2 9 0}$ & $\mathbf{0 , 4 5 5}$ & ND & ND & ND \\
& P.i & ND & 0,086 & 0,012 & 0,034 & 0,028 & 0,086 & 0,031 & ND & ND & ND \\
\hline \multirow{2}{*}{ Citoprotetor } & P.a & $\mathbf{0 , 6 8 0}$ & $\mathbf{0 , 6 5 2}$ & $\mathbf{0 , 5 1 3}$ & $\mathbf{0 , 7 0 2}$ & $\mathbf{0 , 6 9 3}$ & $\mathbf{0 , 6 5 2}$ & $\mathbf{0 , 5 6 9}$ & ND & $\mathbf{0 , 7 0 6}$ & $\mathbf{0 , 6 9 4}$ \\
& P.i & 0,007 & 0,014 & 0,059 & 0,005 & 0,005 & 0,014 & 0,042 & ND & 0,005 & 0,005 \\
\hline
\end{tabular}

Fonte: elaborado pelos autores

Potencial de ser ativo $>50 \%$ : AZUL Potencial de ser ativo $<50 \%$ : VERMELHO

Algumas moléculas não possuíam certas atividades farmacológicas disponíveis na plataforma PASS Online ${ }^{\circledR}(\mathrm{ND})$. A seguir, estão descritas as avaliações que se encontravam indisponíveis, acompanhadas de tais moléculas, respectivamente:

- $\quad$ Retinoprotetor - luteolina;

- Estimulante de eritropoiese - orientina;

- Antimetastático - ácido gálico, luteolina e apigenina.

Conforme descrito nas tabelas 1, 2 e 3, nenhuma avaliação do composto Cianeto-3-0-glucosídeo encontrava-se disponível na plataforma PASS Online ${ }^{\circledR}$. Vale ressaltar que este estudo apresenta, por limitação, a não utilização de outras plataformas de análise computacional, o que pode vir a ser complementado, futuramente, para elucidar e demonstrar, ainda mais, a associação dos efeitos bioativos do extrato de açaí com sua matriz química.

\section{CONCLUSÃO}

De acordo com os resultados obtidos, confirma-se que as moléculas presentes no extrato hidroalcoólico de Euterpe oleracea Mart. (açaí) possuem intensa atividade biológica relacionada ao estresse oxidativo, sendo capazes de atuar a nível de emparelhamento de elétrons, eliminando radicais livres com probabilidade de ser ativo ( $\mathrm{Pa}$ ) acima de 90\%.

No tocante a suas atividades anti-inflamatórias, destaca-se a expressiva supressão de mediadores inflamatórios como o TNF, impedindo que um processo citotóxico se estabeleça com a liberação excessiva da cascata de inflamação, já que as taxas de Pa se mantiveram acima de $70 \%$, quando avaliados quanto ao seu efeito inibidor de TNF. Ainda, na avaliação das propriedades farmacológicas desse fruto, os efeitos benéficos nos cânceres chamam a atenção, visto que em relação aos potenciais anticarcinogênico e antineoplásico houve moléculas com efeitos acima de $80 \%$ e o potencial antimutagênico superou $90 \%$.

Por fim, este estudo de predição de espectro de atividades sugere que, de fato, os efeitos biológicos do açaí, que vêm sendo descritos em estudos in vitro, ex vivo e in vivo, devem-se a sua matriz química, pois ela é composta por uma variedade de moléculas bioativas que podem interagir 
positivamente com células, tecidos e mecanismos diversos. Desse modo, pode ser uma excelente alternativa a estudos que buscam pelo desenvolvimento de novos métodos terapêuticos em geral.

\section{AGRADECIMENTOS}

Agradecemos ao Conselho Nacional de Desenvolvimento Científico e Tecnológico (CNPq) (protocolo número: 423666/2018-5).

\section{REFERÊNCIAS}

ADÃO, R. et al. Cardiotoxicidade associada à terapêutica oncológica: mecanismos fisiopatológicos e estratégias de prevenção. Revista Portuguesa de Cardiologia. v. 32, p. 395-409, 2013.

ANDREAZZA, A. C. et al. Specific subcellular changes in oxidative stress in prefrontal cortex from patients with bipolar disorder. Journal of Neurochemistry. v. 127, p. 552-561, 2013.

BAPTISTA, K. C. R. Avaliação do efeito do açaí (Euterpe oleracea) em modelo de endometriose, e descrição dos dados de toxicidade e do efeito anticancerígeno do açaí em modelos experimentais. 126 f. Dissertação (Mestrado em Saúde Pública e Meio Ambiente) - Escola Nacional de Saúde Pública Sergio Arouca, Fundação Oswaldo Cruz, 2018.

BOCKENHAUER, D.; BICHET, D. G.; Pathophysiology, Diagnosis and Management of Nephrogenic Diabetes Insipidus. Nature Reviews. Nephrology. v. 11, p. 576-588, 2015.

BOLIGON, A. A. et al. HPLC analysis and antimicrobial, antimycobacterial and antiviral activities of Tabernaemontana catharinensis. Journal of Applied Biomedicine. v. 3, p. 7-18, 2015.

CADONÁ, F. C. et al. Guaraná a Caffeine-Rich Food Increases Oxaliplatin Sensitivity of Colorectal HT-29 Cells by Apoptosis Pathway Modulation. Anti-Cancer Agents in Medicinal Chemistry. v. 16, p. 1055-1065, 2016.

CADONÁ, F. C. et al. Guaraná, a Highly Caffeinated Food, Presents in vitro Antitumor Activity in Colorectal and Breast Cancer Cell Lines by Inhibiting AKT/mTOR/S6K and MAPKs Pathways. Nutrition And Cancer-An International Journal, v. 69, p. 1-11, 2017. 
CADONÁ, F. C. et al. Açaí (Euterpe oleracea Mart.) as a Potential Anti-neuroinflammatory Agent: NLRP3 Priming and Activating Signal Pathway Modulation. Mol Neurobiol. 2021. DOI: 10.1007/ s12035-021-02394-x

CROWLEY, L. C.; WATERHOUSE, N. J. Detecting Cleaved Caspase-3 in Apoptotic Cells by Flow Cytometry._Cold Spring Harbor Protocols. 2016 (11) doi: 10.1101/pdb.prot087312.

DeCS - DESCRITORES EM CIÊNCIAS DA SAÚDE. Fator de Necrose Tumoral Alfa. Biblioteca Virtual em Saúde. Disponível em: https://decs.bvsalud.org/ Acesso em: 13/12/2020

DE LIMA, D. Y. Efeitos do extrato de açaí (Euterpe oleracea) sobre o estresse oxidativo e a inflamação em célula mesangial imortalizada de camundongo cultivada em meio com alta concentração de glicose. Plataforma Sucupira. Dissertação de mestrado - UNIFESP. 2017

DE OLIVEIRA, P. R. B. et al. Euterpe oleracea Mart.-Derived Polyphenols Protect Mice from Diet-Induced Obesity and Fatty Liver by Regulating Hepatic Lipogenesis and Cholesterol Excretion. PLoS ONE. 10 (12), 2015.

DE SOUZA, D. V. et al. Açaí (Euterpe oleracea Mart.) presents anti-neuroinflammatory capacity in LPS-activated microglia cells. Nutritional Neuroscience. 2020. doi: 10.1080/1028415X.2020.1842044

DEL POZO-INSFRAN, D.; PERCIVAL, S. S.; TALCOTT, S. T.; Acai (Euterpe oleracea Mart.) Polyphenolics in Their Glycoside and Aglycone Forms Induce Apoptosis of HL-60 Leukemia Cells. Journal of Agricultural and Food Chemistry. v. 54, p. 1222-1229, 2006.

FERLAY, J. et al. Global Cancer Statistics, 2012. CA: Cancer Journal for Clinicians. v.65, n. 2, p. 87-108, 2015.

FONSECA, L. X. et al. Caracterização química e física de frutos de butiá (Butia capitata). In: XIII Congreso Argentino de Ciencia y Tecnologia de Alimentos. Buenos Aires, v. 1. p. 1-1. 2011.

GALlEANO, M.; PECHANOVA, O.; FRAGA, C. G. Hypertension, nitric oxide, oxidants, and dietary plant polyphenols. Current Pharmaceutical Biotechnology. v. 11, p. 837-848, 2010.

GENTILE D. et al. Luteolin Prevents Cardiometabolic Alterations and Vascular Dysfunction in Mice With HFD-Induced Obesity. Frontiers in Pharmacology. v. 9, article 1094, 2018. 
GRUNZE, H. et al. The World Federation of Societies of Biological Psychiatry (WFSBP) guidelines for the biological treatment of bipolar disorders: update 2010 on the treatment of acute bipolar depression. The World Journal of Biological Psychiatry. v. 11, n. 2, p. 81-109, 2010.

HARBONE, J. B.; WILLIAMS, C. A. Advances in flavonoid research since 1992. Phytochemistry. v. 55 , p. 481-504, 2000.

LAJOLO, F. M. Alimentos funcionais: aspectos científicos e normativos. Dieta e saúde. 2002.

LOBO, A. C. M.; VELASQUE, L. F. L. Revisão de literatura sobre os efeitos terapêuticos do açaí e sua importância na alimentação. Biosaúde. Londrina. v. 18, n. 2, 2016.

MACHADO, A. K. et al. Guaraná (Paullinia cupana) improves the proliferation and oxidative metabolism of senescent adipocyte stem cells derived from human lipoaspirates. Food Research International. v. 67, p. 426-433, 2014.

MACHADO, A. K. et al. Neuroprotective Effects of Açaí (Euterpe oleracea Mart.) against Rotenone In Vitro Exposure. Oxidative Medicine and Cellular Longevity. v. 2016, article ID 8940850.

MACHADO, A. K. et al. Upstream pathways controlling mitochondrial function in major psychosis: a focus on bipolar disorder. The Canadian Journal of Psychiatry. v. 61, p. 446-456, 2016.

MACHADO, A. K. et al. Açaí (Euterpe oleracea Mart.) has anti-inflammatory potential through NLRP3-inflammasome modulation. Journal of Functional Foods. v. 56, p. 364-371, 2019.

MANCINI, M. C. Obstáculos diagnósticos e desafios terapêuticos no paciente obeso. Arquivos Brasileiros de Endocrinologia e Metabologia. v. 45, p. 584-608, 2001.

MINISTÉRIO DA SAÚDE. Brasil. Secretaria de Atenção à Saúde. Departamento de Atenção Básica. Guia alimentar para a população brasileira. 2. ed., 1. reimpr. - Brasília, Ministério da Saúde, 2014.

NEWMAN, D. J.; CRAGG, G. M. Natural Products as Sources of New Drugs from 1981 to 2014. Journal of Natural Products. v. 79, p. 629-661, 2016.

NUSSBAUM, R. L. et al. Thompson \& Thompson Genética Médica. Genômica e Genética do Câncer. 7. ed. São Paulo: Elsevier, 2008. p. 1204-1219. 
PORTINHO, J. A.; ZIMMERMANN, L. M.; BRUCK, M. R. Beneficial effects of açaí. International Journal of Nutrology. v. 5, n. 1, p. 15-20, 2012.

RESENDE, F. A. Estudo do potencial antimutagênico, mutagênico, estrogênico e antibacteriano de flavonoides. 2011. 181 f. Tese (doutorado) - Universidade Estadual Paulista, Faculdade de Ciências Farmacêuticas, 2011. Disponível em: http://hdl.handle.net/11449/104031.

ROCHA, A. P. M. et al. Endothelium-dependent vasodilator effect of Euterpe oleracea Mart. (Açai) extracts in mesenteric vascular bed of the rat. Vascul Pharmacol. v. 46(2), p. 97-104, 2007.

SAGRILLO, M. R. et al. Tucumã fruit extracts (Astrocaryum aculeatum Meyer) decrease cytotoxic effects of hydrogen peroxide on human lymphocytes. Food Chemistry. v. 173, p. 741-748, 2015.

WANG, D. et al. Clinical characteristics of 138 hospitalized patients with 2019 novel coronavirus-infected pneumonia in Wuhan, China. JAMA - Journal of the American Medical Association. v. 323, p. 1061-1069, 2020. 